\title{
Simulation of Cavity Flow by the Lattice Boltzmann Method
}

\author{
Shuling Hou ${ }^{1,3}$, Qisu Zou ${ }^{2}$, Shiyi Chen ${ }^{1}$, Gary D. Doolen ${ }^{1}$, Allen C. Cogley ${ }^{3}$ \\ ${ }^{1}$ Center for Nonlinear Studies and Theoretical Division, Los Alamos National Laboratory, \\ Los Alamos, NM 87545 \\ ${ }^{2}$ Department of Mathematics, Kansas State University, Manhattan, KS 66506. \\ ${ }^{3}$ Department of Mechanical Engineering, Kansas State University, Manhattan, KS 66506.
}

\begin{abstract}
A detailed analysis is presented to demonstrate the capabilities of the lattice Boltzmann method. Thorough comparisons with other numerical solutions for the two-dimensional, driven cavity flow show that the lattice Boltzmann method gives accurate results over a wide range of Reynolds numbers. Studies of errors and convergence rates are carried out. Compressibility effects are quantified for different maximum velocities, and parameter ranges are found for stable simulations. The paper's objective is to stimulate further work using this relatively new approach for applied engineering problems in transport phenomena utilizing parallel computers.
\end{abstract}




\section{Introduction}

Lattice gas automata (LGA) and its later derivative, the lattice Boltzmann equation method (LBE), are relatively new approaches that utilize parallel computers to study transport phenomena. Since the first two-dimensional model representing incompressible Navier-Stokes equations was proposed by Frisch, Hasslacher, and Pomeau (FHP) in 1986 [1], LGA have attracted much attention as promising methods for solving a variety of partial differential equations and modeling physical phenomena $[2,3,4,5]$.

A lattice gas is constructed as a simplified, fictitious microworld in which space, time and velocities are all discrete. In general, a lattice gas consists of a regular lattice with particles residing on the nodes. A set of Boolean variables $n_{i}(\mathbf{x}, t)(i=1, \cdots, b)$ for describing the particle occupation is defined, where $b$ is the number of directions of particle velocity at each node. Starting from an initial state, the configuration of particles at each time step evolves in two sequential steps: (a) streaming, where each particle moves to the nearest node in the direction of its velocity; and (b) colliding, which occurs when particles arriving at a node interact and possibly change their velocity directions according to scattering rules. For simplicity, the exclusion principle (no more than one particle is allowed at a given time and node moving in a given direction) is imposed for memory efficiency and leads to the FermiDirac equilibrium distribution. The strategy of the lattice gas is two-fold: a) to construct a model as simple as possible of the microworld to permit simulations of a system composed of many particles and b) to capture the essential features of real collision processes between particles such that, for long times and large scales, macroscopic transport phenomena are captured.

That the evolution of particles on an artificial lattice can simulate the macroscopic 
behavior of fluid flow is based on the following facts: the macro-dynamics of a fluid is the result of the collective behavior of many particles in the system and details of the microscopic interactions are not essential. Changes in molecular interactions affect transport properties such as viscosity, but do not alter the basic form of the macroscopic equations as long as the basic conservation laws and necessary symmetries are satisfied $[2,3]$.

Due to the microscopic nature and local interaction between particles, the lattice gas approach possesses some unique advantages. The scheme is absolutely stable; boundary conditions are easy to implement; the model is ideal for massively parallel computing and the code is simple. The lattice gas method also contains some problems such as nonGalilean invariance, due to the existence of a density-dependent coefficient in the convection term of the Navier-Stokes equation, an unphysical velocity-dependent pressure and inherent statistical noise that requires a spatial (or time) averaging to obtain smooth macroscopic quantities. To avoid some of these inherent problems, several lattice Boltzmann (equation) models have been proposed $[6,7,8,9,10,11]$. The main feature of the LBE is to replace the particle occupation variables, $n_{i}$, (Boolean variables) by the single-particle distribution functions (real variables) $f_{i}=\left\langle n_{i}\right\rangle$, where \langle\rangle denotes a local ensemble average, in the evolution equation, i.e. the lattice Boltzmann equation.

The lattice Boltzmann equation as a numerical scheme was first proposed by McNamara and Zanetti [6]. In their model, the collision operator is the same as in the LGA. Higuera, Jimenez and Succi $[7,8]$ introduce a linearized collision operator that is a matrix and has no correspondence to the detailed collision rules. Statistical noise is completely eliminated in both models; however, the other problems remain since the equilibrium distribution is still Fermi-Dirac. The lattice Boltzmann model proposed by Chen et al. $[9,11]$ and Qian et al. 
[10] abandons Fermi-Dirac statistics and provides the freedom required for the equilibrium distribution to satisfy isotropy, Galilean invariance and to possess a velocity-independent pressure. Their models apply the single relaxation time approximation first introduced by Bhatnager, Gross and Krook in 1954 [12] to greatly simplify the collision operator. This model is called the lattice Boltzmann BGK model.

Compared with the lattice gas approach, the lattice Boltzmann method is more computationally efficient using current parallel computers. Applications have been done using both methods on hydrodynamics $[13,14,15,16]$, flow through porous media $[17,18]$, magnetohydrodynamics $[19,20]$, multiphase flow $[21,22,23,24]$ and the reaction-diffusion equation $[25,26,27]$. Collected papers and applications of lattice gas and lattice Boltzmann methods can be found in $[4,5,28,29]$.

Despite these studies on various problems, thorough quantitative investigations of the method have not been published. In the present work, the lattice Boltzmann BGK model (LBGK) is used to solve for the viscous flow in a square, two-dimensional cavity driven by shear from a moving wall for Reynolds numbers up to 10,000. Detailed comparisons between the LBGK and traditional methods are presented. The compressibility error and the convergence rate of the method are discussed. The objective of this paper is to analyze the accuracy and physical fidelity of the lattice Boltzmann BGK method and to stimulate further studies using the lattice Boltzmann approach.

Section 2 presents a technical synopsis of the lattice Boltzmann model used in this paper that will enhance the general reader's understanding of this simulation method. More technical details are given in the Appendix for those who want to use the lattice Boltzmann method. The lattice Boltzmann simulation of driven cavity flow is discussed in Section 3 
and thoroughly compared with results from other numerical methods. Section 4 studies the numerical errors in lattice Boltzmann simulations due to lattice size and compressibility. Section 5 is devoted to comparisons between the square lattice and the triangular (FHP) lattice. The limit of relaxation time for these two models is explored. The final section contains concluding remarks.

\section{Two-Dimensional Square Lattice Boltzmann Model}

In this section an outline is given of the procedures of the lattice Boltzmann simulation. A square lattice with unit spacing is used on which each node has 8 nearest neighbors connected by 8 links (see Figure $1 \mathrm{~A}$ in the Appendix). Particles can only reside on the nodes and move to their nearest neighbors along these links in the unit time step. Hence, there are two types of moving particles. Particles of type 1 move along the axes with speed $\left|\mathbf{e}_{\mathbf{1}}\right|=1$ and particles of type 2 move along the diagonal directions with speed $\left|\mathbf{e}_{\mathbf{2}}\right|=\sqrt{2}$. Rest particles with speed zero are also allowed at each node. The occupations of the three types of particles is represented by the single-particle distribution function, $f_{\sigma i}(\mathbf{x}, t)$, where subscripts $\sigma$ and $i$ indicate the type of particle and the velocity direction, respectively. The distribution function, $f_{\sigma i}(\mathbf{x}, t)$, is the probability of finding a particle at node $\mathbf{x}$ and time $t$ with velocity $\mathbf{e}_{\sigma i}$. The particle distribution function satisfies the following lattice Boltzmann equation:

$$
f_{\sigma i}\left(\mathbf{x}+\mathbf{e}_{\sigma i}, t+1\right)-f_{\sigma i}(\mathbf{x}, t)=\Omega_{\sigma i}
$$

where $\Omega_{\sigma i}$ is the collision operator representing the rate of change of the particle distribution

due to collisions. According to Bhatnagar, Gross and Krook (BGK) [12], the collision operator is simplified by the single time relaxation approximation. Hence, the lattice Boltzmann 
BGK equation is

$$
f_{\sigma i}\left(\mathbf{x}+\mathbf{e}_{\sigma i}, t+1\right)-f_{\sigma i}(\mathbf{x}, t)=-\frac{1}{\tau}\left[f_{\sigma i}(\mathbf{x}, t)-f_{\sigma i}^{(0)}(\mathbf{x}, t)\right]
$$

where $f_{\sigma i}^{(0)}(\mathbf{x}, t)$ is the equilibrium distribution at $\mathbf{x}, t$ and $\tau$ is the single relaxation time which controls the rate of approach to equilibrium. The density per node, $\rho$, and the macroscopic velocity, $\mathbf{u}$, are defined in terms of the particle distribution function by

$$
\sum_{\sigma} \sum_{i} f_{\sigma i}=\rho
$$

and

$$
\sum_{\sigma} \sum_{i} f_{\sigma i} \mathbf{e}_{\sigma i}=\rho \mathbf{u}
$$

The equilibrium distribution can be chosen in the following form for particles of each type:

$$
\begin{aligned}
f_{01}^{(0)} & =\frac{4}{9} \rho\left[1-\frac{3}{2} u^{2}\right], \\
f_{1 i}^{(0)} & =\frac{1}{9} \rho\left[1+3\left(\mathbf{e}_{1 i} \cdot \mathbf{u}\right)+\frac{9}{2}\left(\mathbf{e}_{1 i} \cdot \mathbf{u}\right)^{2}-\frac{3}{2} u^{2}\right], \\
f_{2 i}^{(0)} & =\frac{1}{36} \rho\left[1+3\left(\mathbf{e}_{2 i} \cdot \mathbf{u}\right)+\frac{9}{2}\left(\mathbf{e}_{2 i} \cdot \mathbf{u}\right)^{2}-\frac{3}{2} u^{2}\right] .
\end{aligned}
$$

The relaxation time is related to the viscosity by

$$
\nu=\frac{2 \tau-1}{6}
$$

where $\nu$ is the kinematic viscosity. The detailed derivation of the LBGK model is given in the Appendix.

Having chosen the appropriate lattice size and the characteristic velocity for the LBE system, the viscosity, $\nu$, of the problem can be calculated for a given Re number and then the relaxation time is determined by the formula above. Starting from an initial state of $f_{\sigma i}(\mathbf{x}, t)$, the density and velocity fields and hence the equilibrium distribution function 
can be obtained. In each time step, the updating of the particle distribution can be split into two substeps: collision and streaming. It is irrelevant which one is the first for a long time run. The collision process at position $\mathbf{x}$ occurs according to the right hand side of the Boltzmann equation given as Eq. (2). The resulting particle distribution at $\mathbf{x}$, which is the sum of the original distribution and the collision term, is then streamed to the nearest neighbor of $\mathbf{x}, \mathbf{x}+\mathbf{e}_{\sigma i}$, according to the particle velocity $\mathbf{e}_{\sigma i}$. The updating procedure is terminated for steady state problems when certain criteria are reached. The method can be used for transient problems, but this will not be discussed in this paper.

The boundary condition commonly used at the solid wall of a fluid simulation is the no-slip condition for which the velocities vanish at the wall. This is implemented in the lattice gas and lattice Boltzmann methods with the bounce-back rule in which all particles hitting the wall are reflected back in the direction from which they came.

Another lattice model commonly used in two-dimensional lattice gas and lattice Boltzmann simulations is the triangular lattice (FHP model) $[1,2]$. This is a two-speed (0 and 1) model in which the lattice constant (link) is equal to one. Simulations of cavity flow are also performed in this paper using this model. Comparisons between FHP and square lattice are discussed in Section 5. Two commonly used models for three-dimensional simulations are the 24-velocity FCHC [2] and the 14-velocity cubic models [10, 14].

\section{Cavity Simulation}

The problem considered is two-dimensional viscous flow in a cavity governed by the NavierStokes equations. An incompressible fluid is bounded in a square enclosure and the flow is driven by the uniform translation of the top boundary. The fluid motion generated in 
a cavity is an example of closed streamline problems that are of theoretical importance because they are part of broader field of steady, separated flows. The literature is abundant for this flow configuration that shows rich vortex phenomena at many scales depending on the Reynolds number, Re. Numerical methods for solving the Navier-Stokes equations are often tested and evaluated on cavity flows because of the complexity of the flows.

Most numerical solutions of two-dimensional cavity flow [32-40] use a vorticity-stream function formulation and discretize the incompressible, steady linear or nonlinear NavierStokes equations by finite difference [32-35], multigrid [36, 38, 39] and finite element [40] methods and their variations [37]. Earlier work was reviewed by O. Burggraf [32] where his numerical solutions of the nonlinear Navier-Stokes equations for Reynolds number up to 400 showed a large primary vortex and two secondary vortices in the lower corners. The later studies of A. S. Benjamin and V. E. Denny [35], U. Ghia, K. N. Ghia and C. T. Shin [36], R. Schreiber and H. B. Keller [37] show that tertiary vortices are formed near the bottom corners for higher Reynolds numbers. The present results using the lattice Boltzmann method are compared with those done by Vanka [38], Schreiber and Keller [37], Ghia et al. [36] and Zhou et al. [39]. Ghia et al. obtained numerical solutions up to Re=10,000 with a $257 \times 257$ grid using the coupled strongly implicit multigrid method and vorticity-stream function formulation. Their work is the most comprehensive study of cavity flow to date.

The present simulation uses Cartesian coordinates with the origin located at lower left corner. The top wall is moving from left to right with velocity $U$. The cavity has 256 lattice units on each side. Initially the velocities at all nodes, except the top, are set to zero. The x-velocity of the top is $U$ and the y-velocity is zero. Uniform initial particle density is imposed such that the moving particle 1 has a density fraction of $d=\frac{\rho}{9}=0.3$ per direction. 
The moving particle 2 has a density fraction of $\frac{d}{4}$ per direction, and the rest particle has a density of $4 d$. Therefore, the total density per node is $\rho=2.7$. Using the uniform density distribution and velocities given above, the equilibrium particle distribution function, $f_{i}$, is calculated according to Eq. (5). The evolution of $f_{i}$ can then be found by a succession of streaming and collision-like processes. After streaming, the velocity of the top lid is reset to the uniform initial velocity. At the end of each streaming and collision process cycle, the particle distribution function, $f_{i}$, at the top is set to the equilibrium state and the bounce-back boundary conditions are used on the three stationary walls. The two upper corners are singular points which are considered as part of the moving lid in the simulations, but tests shown there is little difference if these two points are treated as fixed wall points. The uniform velocity of the top wall used in the simulations is $U=0.1$. The compressibility effects are discussed in Section 4.4. The Reynolds number used in the cavity simulation is defined as $R e=\mathrm{U} L / \nu$, where $U$ is the uniform velocity of the top plate, $L$ is the edge length of the cavity and $\nu$ is the kinematic viscosity that is related to the single relaxation time as given in Eq. (6). All the results are normalized to allow comparisons between the present work and other results based on a unit square cavity with unit velocity of the top boundary.

Steady-state solutions for cavity flow are obtained using the lattice Boltzmann method for $\operatorname{Re}=10,100,200,400,1,000,2,000,5,000$, and 7,500. The $\operatorname{Re}=10,000$ case is also run on a $256 \times 256$ lattice, but steady state cannot be reached because bifurcation takes place somewhere between $\operatorname{Re}=7,500$ and 10,000. The results for $\operatorname{Re}=10,000$ oscillate between a series of different configurations. For this reason the results presented in this paper are those for Re up to 7,500. The dependent variables of stream function, velocity, pressure 
and vorticity are calculated using the particle distribution function, $f_{i}$. The dependent parameter of the drag coefficient of the driving wall is discussed also.

\subsection{Stream function}

Figure 1 (a-g) shows plots of the stream function for the Reynolds numbers considered. It is apparent that the flow structure is in good agreement with the previous work of Benjamin and Denny [35], Schreiber and Keller [37] and Ghia et al. [36]. These plots give a clear picture of the overall flow pattern and the effect of Reynolds number on the structure of the steady recirculating eddies in the cavity. In addition to the primary, center vortex, a pair of counterrotating eddies of much smaller strength develop in the lower corners of the cavity at higher values of Re. At $\operatorname{Re}=2000$, a third secondary vortex is seen in the upper left corner (it is generated at a critical Re of about 1,200 according to [35] and that agrees with the results of the present work). For $\operatorname{Re} \geq 5,000$, a tertiary vortex in the lower right hand corner appears. A series of eddies with exponentially decreasing strength in the lower corners has been predicted [36]. Due to the compressibility effect of the LBE (discussed in Section 4.4), the tertiary vortex shown in the Figure 1 (g) oscillates.

For low $\operatorname{Re}($ e.g. $\operatorname{Re}=10)$, the center of the primary vortex is located at the midwidth and at about one third of the cavity depth from the top (see Figure 1.a). As Re increases $(\operatorname{Re}=100)$, the primary vortex center moves towards the right and becomes increasing circular. Finally, this center moves down towards the geometric center of the cavity as the Re increases and becomes fixed in its $\mathrm{x}$ location for $\mathrm{Re} \geq 5,000$. The movement of the vortex center location versus Re is shown in Figure 2 along with the results given by Ghia et al. [36]. 
To quantify these results, the maximum stream function value for the primary vortex and the minimum values for the secondary vortices along with the $\mathrm{x}$ and $\mathrm{y}$ coordinates of the center of these vortices are listed in Table 1. All the results presented use a uniform top velocity $U=0.1$ except for $\mathrm{Re}=100$ where $\mathrm{U}=0.01$ is used. The reason is discussed in Section 4.4. Also listed are results selected from previous work [36-39]. Previous results agree with each other for $\operatorname{Re} \leq 1000$, but vary for higher values of Re. The results of the present work and that of Ghia et al. [36] for stream function values agree within $0.2 \%$ for all values of $\operatorname{Re}(\operatorname{Re}=2,000$ data was not given by [36]). The locations of the vortex centers predicted by the lattice Boltzmann method also agree well with those given by Ghia et al. $[36]$.

Unlike finite-difference or finite-element methods that start from the steady-state, partial differential equations, the present method is a direct modeling method that evolves into steady state. The time to reach steady-state depends on the lattice size, the values of Re and the driving velocity, $U$. For all the cases run in this paper, steady-state is reached when the difference between the maximum values of the stream function for successive 10,000 steps (process cycles) is less than $10^{-5}$. Considering the kinetic, direct, compressible and unsteady nature of the lattice Boltzmann method, the excellent agreement with entirely different methods such as Ghia et al. [36] is quite encouraging.

The minimum values of stream function and the center of the secondary vortex in the upper left corner for $\mathrm{Re}=5,000$ and 7,500 are listed in Table 2. These results also show good agreement with Ghia et al. [36]. 


\section{$3.2 \quad$ Velocity profiles}

Velocity components along a vertical and horizontal center line for several values of Re are shown in Figure 3. The velocity profiles change from curved at lower values of Re to linear for higher Re values. The near linear profiles of the velocity in the central core of the cavity indicate the uniform vorticity region generated in the cavity at higher values of Re. These results agree with those from previous studies [34, 36, 37].

\subsection{Vorticity}

The plots of vorticity in Figure 4 (a-g) show that the steady cavity flow within closed streamlines at high Re consists of a central, inviscid core of nearly constant vorticity with viscous effects confined to thin shear layers near the walls. Batchelor [30] predicted these results from his model for separated eddies in a steady flow. As the Re increases, several regions of high vorticity gradients (indicated by concentration and wiggle of the vorticity contours) appear within the cavity. The thinning of the wall boundary layers with increasing Re is evident from these plots, although the rate of this thinning is very slow for $\operatorname{Re} \geq 5,000$. The values of vorticity at the center of the primary vortex for different Re are listed in Table 3. These values closely agree with the results of Ghia et al. [36] and approach the analytical value of 1.886 for an infinite Reynolds number calculated by Burggraf [32] using Batchelor's model.

\subsection{Pressure}

Figure $5(\mathrm{a}-\mathrm{g})$ displays the pressure deviation contours for the present simulations. Since only the pressure gradient appears in the Navier-Stokes equation, the values of pressure 
can deffer linearly. These plots are in good agreement with the static pressure given by Burggraf [32] (note that the top wall in [32] moves from right to left which is opposite to that in the present simulation). The pressure in [32] is obtained by integrating the NavierStokes equation given the velocity field, while the pressure in the lattice Boltzmann method satisfies the equation of state of the isothermal gas where it is proportional to the density. The observed agreement between these very different approaches demonstrates that the lattice Boltzmann BGK model is valid for simulating incompressible flow. By examing the closed contours in the pressure plots, it is seen that the inviscid core grows with increasing values of Re. In the opposite limit of Re approaching zero, pressure becomes a harmonic function and the contours cannot be closed but must end on the boundaries [32]. The present results support this last requirement.

\subsection{Drag on the top}

The drag force and the drag coefficient of the moving wall is calculated here for Re values considered in the study. The stress on the moving wall is given by the Newton's formula as

$$
\tau_{y x}=\mu \frac{\partial u}{\partial y}
$$

where $u$ is the $\mathrm{x}$ component of velocity and $\mu$ is the kinetic viscosity. The drag force on this surface, $F_{d}$, is defined as

$$
F_{d}=\int_{0}^{L} \tau_{y x} d x=, \int_{0}^{L} \mu \frac{\partial u}{\partial y} d x=\mu \sum_{i=2}^{i=n_{x}-1} \frac{u(i, 2)-u(i, 1)}{\triangle y} \triangle x,
$$

where $n_{x}$ is the grid number in the $\mathrm{x}$ direction, $L$ is the length of the square cavity and $\triangle y=\triangle x=\frac{L}{\left(n_{x}-1\right)}$ are the spacing of the lattice. The drag coefficient is then written as

$$
C_{d}=\frac{F_{d}}{\bar{\rho} U^{2} L}
$$


where $\bar{\rho}$ is the average density and $U$ is the velocity of the top. The drag coefficient decreases as Re increases, as found in other laminar flow configurations. This can be seen by introducing the dimensionless quantities

$$
u^{\prime}=\frac{u}{U}, \quad x^{\prime}=\frac{x}{L}, \quad y^{\prime}=\frac{y}{L}
$$

The drag coefficient can then be expressed as

$$
C_{d}=\frac{U \int_{0}^{1} \mu \frac{\partial u^{\prime}}{\partial y^{\prime}} d x^{\prime}}{\bar{\rho} U^{2} L}=\int_{0}^{1} \frac{\mu}{\bar{\rho} U L} \frac{\partial u^{\prime}}{\partial y^{\prime}} d x^{\prime}=\frac{1}{R e} \int_{0}^{1} \frac{\partial u^{\prime}}{\partial y^{\prime}} d x^{\prime} .
$$

The results of drag and drag coefficient for different values of Re are listed in Table 4 and the latter is plotted in Figure 6. The plot shows that the relation between drag coefficients and Re satisfies the above formula. There is no data available on drag and drag coefficient from other methods for comparison.

\section{Error Analysis}

\subsection{Sources of errors}

There is no analytic solution for cavity flow. Results from the work described in this paper are compared with the numerical solutions obtained by several other methods. Differences are found between the results of previous work, especially for higher values of Re. Several of these authors state that the data for the secondary vortices are less reliable due to corner singularities and/or roundoff errors [37], mesh-size limitations [34] or because the values of the stream function in the corners are small and, in some cases, below the convergence accuracy of the calculations [38].

Before the final results listed in Table 1 were obtained, the results from lattice Boltzmann simulations were very close to the results given by Ghia et al. [36] for $\operatorname{Re} \geq 1,000$ only. The 
properties of the secondary vortices were less satisfactory for Re less than 1,000. The secondary vortex of the lower left corner for $\mathrm{Re}=100$, whose stream function is a small quantity of the order of $10^{-6}$, was not detected by the lattice Boltzmann method used in the initial simulations. Also, the secondary vortex in the lower right corner for the same Re, whose stream function is of the order of $10^{-5}$, did not match corresponding results of other investigators. Although these are not major features, it was important to investigate the cause of these discrepancies.

The theoretical assumptions of the present method are the Boltzmann transport equation plus the single relaxation time approximation of the collision term. As long as the macroscopic properties of fluid vary slowly enough in space and time compared with microscopic particle dynamics, collisions should maintain approximately the local equilibrium such that the assumptions of molecular chaos by Boltzmann and single relaxation time by BGK are valid for problems of fluid dynamics. The possible reasons for the "errors" in the present simulations may be categorized as follows:

1. The simulations done for different values of $\operatorname{Re}$ on a $256 \times 256$ lattice used single precision arithmetic. It is possible that roundoff error could be accumulated.

2. The small compressibility effect presented in the LBE simulations may cause differences when compared with models where compressibility is zero.

3. The lattice size used here may still be too coarse to resolve all the small scale phenomena.

4. The time step at which the simulation is terminated may not be large enough to represent steady state.

5. The integration methods used in the calculation of the stream function may introduce 
errors.

The errors caused by 1 . and 4. can be avoided by using double precision floating-point arithmetic and running for longer times. These experiments did not change the result for weak vortices on the smaller scales. What follows are subsections investigating the rest of the error sources.

\subsection{Effect of lattice size}

To test the effect of lattice size, simulations for $\mathrm{Re}=1,000$ are done on the following lattice configurations: $33 \times 33,65 \times 65,129 \times 129$, and $513 \times 513$. The driving velocity used is kept at $U=0.1$. Two relative velocity errors were calculated according to the following formula:

$$
\begin{gathered}
E 1=\frac{\sum_{x, y}\left|u_{1}-u_{0}\right|+\left|v_{1}-v_{0}\right|}{\sum_{x, y}\left|u_{0}\right|+\left|v_{0}\right|}, \\
E 2=\frac{\sqrt{\sum_{x, y}\left(u_{1}-u_{0}\right)^{2}+\left(v_{1}-v_{0}\right)^{2}}}{\sqrt{\sum_{x, y}\left(u_{0}\right)^{2}+\left(v_{0}\right)^{2}}},
\end{gathered}
$$

where $u, v$ are the $x$ and $y$ components of the velocity, respectively. The subscript 0,1 indicate the $513 \times 513$ and the coarser grain lattice, respectively. Velocities on different grids are taken at corresponding positions, while the sums are taken over the entire lattice. E1 and E2 are two common relative velocity errors in the $L 1$ and $L 2$ norm sense, respectively, based on the highest resolution lattice (513).

The results for $E 1$ and $E 2$ are plotted logarithmically in Fig. 7 and listed in Table 5. The quantities $E 1$ and $E 2$ are calculated also according to the formula above where the 0 and 1 indicate two successive lattice sizes. The results are similar to that shown in Fig. 7. It is clear from Fig. 7 that the convergence rate is approximately first order in space. This 
result is different from other works $[15,41]$ where a second-order convergence rate is claimed. In $[15]$ the problem is a decaying Taylor vortex flow with periodic boundary conditions (no solid wall) and in [41] exact boundary conditions for the particle distribution function are used instead of the bounce-back condition implemented at the wall in the present work. The first order convergence rate observed here may be due to the bounce-back condition used on the stationary walls. This observation is confirmed by a recent paper [42] in which the bounce-back boundary condition is shown to be a first-order approximation for a no-slip wall. It is shown also in [42] that higher order accuracy can be achieved by improving the implementation of boundary conditions.

Better resolution is obtained as the number of lattice nodes increases. However, computer time grows with lattice number because more nodes are updated and the time to reach steady-state is much longer. The time steps required to reach steady state for different lattice sizes are listed in Table 6 . Beyond these facts, a simulation run on a $513 \times 513$ lattice for $\mathrm{Re}=100$ did not improve the method's ability to predict the secondary vortex in the lower, left corner.

\subsection{Integration error}

The most important features of the cavity flow are related to the stream function. The stream function used by Ghia et al. [36] (and others) was the primary variable of the solution. In the lattice Boltzmann model, however, the primary variable is the particle distribution function, $f_{i}$. The velocity at each site is calculated from $f_{i}$ and the stream function is obtained by integrating the velocities.

To investigate the error caused by integration, three integration rules (rectangular, 
trapezoidal and Simpson) are used for $\mathrm{Re}=100$. The results from all three rules are of the same order of accuracy if the integrations are taken in the same direction (of course the trapezoidal and Simpson rules are higher-order integration schemes than the rectangular rule and hence produce less error). Significantly different results are obtained by integrating from the four different directions, namely integrate $u$ along $y$ from top to bottom, integrate $u$ along $y$ from bottom to top, integrate $v$ along $x$ from left to right and integrate $v$ along $x$ from right to left. Theoretically, they should all give the same value for the stream function.

From a numerical point of view, the integration should be taken from the smaller scale, otherwise the smaller scale would be dawn into roundoff error. However, in this particular case, integration from bottom to top contains significant error. The reason can be seen from the error formula. If the trapezoidal rules is used:

$$
I(f)=\int_{a}^{b} f(x) d x \approx h\left[\frac{1}{2} f(a)+f(a+h)+\cdots+\frac{1}{2} f(b)\right]=I_{n}(f)
$$

where $h=\frac{b-a}{n}$ and $n$ is the number of subdivisions within $[a, b]$, the integration error can be expressed as

$$
E_{n}(f)=I(f)-I_{n}(f)=-\frac{h^{2}(b-a)}{12} f^{\prime \prime}\left(c_{n}\right)
$$

Here $c_{n}$ is a point between $a$ and $b$. The above formula can be improved by the asymptotic error formula [43]:

$$
E_{n}(f) \approx-\frac{h^{2}}{12}\left[f^{\prime}(b)-f^{\prime}(a)\right]
$$

The factor, $\frac{h^{2}}{12}$, in the present case is about $1.3 \times 10^{-6}$. The error then depends on the derivatives at the end points. In the case of integration taken from top to bottom or from bottom to top, the two derivatives have opposite signs and the error is enhanced. In addition, the value of the derivative on the top is large. Numerical tests show that integration 
from top to bottom gives inaccurate values $\left(1.96 e^{-3}\right.$ and $1.66 e^{-3}$ for the minimum stream function on the lower left and right corners, respectively). The integration from the bottom to the top predicts another vortex of order $10^{-3}$ on the upper left corner that does not exist for $\mathrm{Re}=100$ and is caused by errors (due to both integration and compressibility). On the other hand, integration from left to right or from the right to left gives the same signs on the two end derivatives, therefore decreasing the integration error. Since the left corner vortex is smaller than that on the right, the integration taken from left to right gives better results $\left(-1.76 e^{-6}\right.$ on left and $-1.10 e^{-5}$ on right) than the integration taken from the right to the left $\left(-2.4 e^{-5}\right.$ on left and $-1.13 e^{-5}$ on right $)$. The conclusion is that using a trapezoidal rule and integrating the velocity component, $v$, from left to right gives the most accurate integral. The error is then of order of $10^{-9}$. The Simpson rule has about the same accuracy.

\subsection{Compressibility effect}

It has been shown that the present LBE model represents the Navier-Stokes equation in the incompressible limit (see Appendix). But in the LBE simulation, the density cannot be a constant (otherwise pressure change cannot be described). It is important to find the effect of compressibility on the present solution.

One quantity that represents compressibility is the mean variation of density. The mean density is defined as

$$
\bar{\rho}=\frac{\sum_{i} \rho\left(x_{i}, t\right)}{N}
$$

where $N$ is the total number of nodes. The mean variation of density is given by

$$
\triangle=\frac{1}{\bar{\rho}} \sqrt{\frac{\sum\left((\rho-\bar{\rho})^{2}\right)}{N}} .
$$


For $\operatorname{Re}=100$, this mean density fluctuation, $\triangle$, is calculated for $U=0.1, U=0.05, U=0.01$ and listed in Table 7 along with the Mach number, $M=\frac{u}{c_{s}}$, where $c_{s}=\frac{1}{\sqrt{3}}$ is the speed of sound for the present model. The table shows that

$$
\triangle(U=0.05) \approx \frac{1}{4} \triangle(U=0.1)
$$

and

$$
\triangle(U=0.01) \approx \frac{1}{25} \triangle(U=0.05)
$$

These results agree with the known relationship [16] that $\triangle$ is proportional to $M^{2}$. (This relation can be seen from the dimensionless incompressible Navier-Stokes equations.)

The compressibility effect can also be examined for the cavity flow problem as follows. In the steady case, the continuity equation represented by LBE is

$$
\nabla \cdot(\rho \mathbf{u})=0
$$

due to a non-constant $\rho$. The velocity $\mathbf{u}$ does not satisfy the incompressible continuity condition given by

$$
\nabla \cdot \mathbf{u}=0
$$

It is from this equation that the stream function can be defined using $u=\frac{\partial \psi}{\partial y}$ and $v=-\frac{\partial \psi}{\partial x}$, where $\psi$ is the stream function. There is actually no exact definition for the stream function in LBE. Given a discrete velocity field obtained from the LBE calculation, an approximation of the stream function for the incompressible flow with $\nabla \cdot(\mathbf{u})=0$ needs to be constructed. The stream function definition written as

$$
\psi=\int-v d x+u d y
$$


is still used to calculate the stream function. When integrating in only the $\mathrm{x}$ - or $\mathrm{y}$-direction, the integral becomes

$$
\psi=\int_{0}^{y} u d y
$$

or

$$
\psi=-\int_{0}^{x} v d x
$$

In the case of incompressible flow in a cavity, the boundaries coincide with the zero stream function. The integrals then take the form

$$
\psi=\int_{0}^{L} u d y=\int_{0}^{L} v d x=0
$$

where $L$ is the total length of the wall. For an incompressible model, integration of $u$ (or $v$ ) along the $y$ (or $x$ ) direction from one edge of the cavity gives a theoretical value of the stream function at another edge of zero. In the actual computations, the stream function at the wall will not exactly equal zero because of roundoff and integration error. Due to the additional effects of compressibility in the LBE method, if the stream function is calculated by integrating $v$ from the left to the right edge of the cavity, the values of the stream function on the right wall would indicate the error caused by compressibility, roundoff and integration errors. Since the trapezoidal rule gives the same results for integrations taken from opposite directions if there is no roundoff error, the roundoff error is found by comparing the values of the stream function on the left and right wall taken from opposite directions (the other sources of error, compressibility and integration, are the same for these two integrals). This error is less than about $10^{-9}$. The integration error is of the order of $10^{-9}$ as discussed above. Therefore, the maximum and the mean value of $\psi$ at the right wall can be computed as an indicator of error due to compressibility if this value is larger than $10^{-8}$. The mean 
and maximum stream function at the right edge of the cavity is defined, respectively, as

$$
S_{a}=\sqrt{\frac{\sum\left(\psi^{2}\left(n_{x}, j\right)\right)}{n_{y}}},
$$

and

$$
S_{m}=\max _{j}\left|\psi\left(n_{x}, j\right)\right|
$$

where $n_{x}=n_{y}=256$ is the number of nodes in the $\mathrm{x}$ - and $\mathrm{y}$-direction, respectively. These values are calculated for $U=0.1, U=0.05$ and $U=0.01$ for $\operatorname{Re}=100$ and listed in Table 8 . Again, the results show that $S_{a}$ and $S_{m}$ are proportional to $M^{2}$. The compressibility error calculated by this argument can be used as a quantitative measure of the compressibility of the LBE method. The change of compressibility error with Re is calculated for $U=0.1$ and listed in Table 9 . The error caused by compressibility does not vary much with Re number. Actually with increased Re the error is slightly decreased, but is still of the same order of magnitude.

It is clear that the error caused by compressibility has about the same order effect as the small scale phenomena in the cavity flow for low values of Re. By choosing the direction of integration for the stream function carefully, predictions of the small vortices are obtained. Furthermore, the compressibility error can be reduced by using smaller velocities at the top wall. The results for $\operatorname{Re}=100$ in Table 1 are calculated using $U=0.01$, while other Reynolds number use $U=0.1$. However, the time steps required to reach steady-state for smaller top velocity increases dramatically as seen in Table 10. To overcome the compressibility error in the present LBE model, a new incompressible LBE model for steady-state flow has been developed and will be published in another paper [44]. 


\section{Triangular Lattice (FHP) Versus Square Lattice}

Simulations for cavity flow are also carried out on a triangular lattice (FHP). There are two types of particles on each node of a FHP model: rest particles and moving particles with unit velocity $\mathbf{e}_{i}$ along 6 directions. In analogy to the procedures used for the square lattice in the Appendix, the equilibrium distributions for the FHP model are given as

$$
\begin{gathered}
f_{0}^{(0)}=d_{0}-\rho u^{2}=\alpha \rho-\rho u^{2} \\
f_{i}^{(0)}=d+\frac{1}{3} \rho\left[\left(\mathbf{e}_{i} \cdot \mathbf{u}\right)+2\left(\mathbf{e}_{i} \cdot \mathbf{u}\right)^{2}-\frac{1}{2} u^{2}\right]=\frac{\rho-\alpha \rho}{6}+\frac{1}{3} \rho\left[\left(\mathbf{e}_{i} \cdot \mathbf{u}\right)+2\left(\mathbf{e}_{i} \cdot \mathbf{u}\right)^{2}-\frac{1}{2} u^{2}\right] .
\end{gathered}
$$

If the ratio of rest and moving particle is defined as

$$
\lambda=\frac{d_{0}}{d},
$$

the pressure is determined by the following isothermal equation of state:

$$
p=3 d=\frac{(1-\alpha) \rho}{2}=\frac{3}{\lambda+6} \rho,
$$

and the speed of sound is

$$
c_{s}^{2}=\frac{1-\alpha}{2}=\frac{3}{\lambda+6}
$$

The viscosity is related to the relaxation time through an equation of the form

$$
\nu=\frac{2 \tau-1}{8}
$$

Theoretically, the relaxation time, $\tau$, cannot be lower than 0.5 for a positive viscosity. To reach higher Re, the relaxation time can be lowed. Tests on a $128 \times 128$ lattice with a maximum velocity of $U=0.1$ show that a critical value for $\tau$ exists. Above this value, the simulation is smooth and reasonable physical patterns for the cavity flow are seen in 
the real-time plots. However, below this critical value, some nonphysical patterns appear. Further reduction in the value of $\tau$ would cause the simulation to be terminated by numerical blow up. Define the critical value of $\tau$ as the lowest limit for the relaxation time that gives physically correct results. This limit varies with the ratio, $\lambda$, maximum velocity, $U$, and the problem studied. If $\lambda$ is increased, the speed of the sound will be decreased and the Mach number is then increased if the velocity is unchanged. Table 11 lists the lowest relaxation time, $\tau_{\min }$, and hence the highest Re number, $R e_{\max }$, obtained for different $\lambda$, along with their speed of sound, $c_{s}$, and Mach number, $M$. Table 11 shows that the highest Re can be increased by increasing $\lambda$. However, the compressibility error is also increased.

Table 12 lists results for the same conditions as in Table 11, but for slightly different initial boundary conditions on the density. In case 1 of Table 11, the initial density on each node of the wall is the same uniform distribution as that for interior nodes. In case 2 of Table 12, the initial density on the wall is set equal to zero. It is clear that when $\lambda=1$, the lowest $\tau$ is much higher than that in Table 11, but for large $\lambda$ the differences of $\tau_{\text {min }}$ between these two cases are diminished. The rest particles in the LBE method play the role of a particle reservoir. When the macroscopic velocity is higher, rest particles can be turned into moving particle and vice versa. Higher values of $\lambda=d_{0} / d$ mean a larger fraction of rest particles in the density behave like a fluid that is less rigid and more flexible (the compressibility is higher). When $\lambda=1$ in case 2 , the lowest value of $\tau$ for stable results is 0.5668 . However, setting $\tau=0.5667$ would make the computation blow up immediately due to the large initial density gradient on the wall and the relatively small fraction of rest particles. The lowest limit of $\tau$ does not depend on the lattice size. Changing the maximum velocity, $U$, does change the lowest $\tau$ slightly. However, the highest Re numbers obtainable 
by this approach are much lower than that for $U=0.1$ (see Table 13 for case 2).

The square lattice corresponds to $\lambda=\frac{d_{0}}{d_{1}}=4$. Tests on a $128 \times 128$ square lattice with the maximum velocity of $U=0.1$ show that the value of $\tau$ cannot be smaller than 0.507 $(\mathrm{Re}=5485)$ for cavity flow. Hence a simulation run on a $256 \times 256$ lattice with $U=0.1$ can reach $\operatorname{Re}=10,000(\tau=0.50768)$ which is about the highest limit of $\operatorname{Re}$ on this size of lattice for cavity flow. Using small $U$ did not produce a further reduction of $\tau$. The square lattice is better than the FHP lattice in the cavity flow simulations because the former can reach higher values of Re than the latter for the same maximum velocity and lattice size. Since the boundaries of the cavity are fitted better using the square lattice than FHP lattice, the formation of the vortices is more gentle in the simulation process using a square lattice than a FHP lattice. The ranges of parameters presented in this section are consistent with the results of linear stability analysis of LBE method without boundaries [45].

\section{Conclusions}

The lattice Boltzmann method is a derivative of the lattice gas automata method and therefore inherits from the LGA some of its advantages over traditional computational methods. It is parallel in nature due to the locality of the transport of particle information, so it is well suited to massively parallel computing. Due to the kinetic description of the lattice Boltzmann method, it is easy to handle the complex boundary conditions and properties of a fluid system, such as flow through porous media and multi-phase flow. One important improvement due to the LBE method is that it can fully recover the Navier-Stokes equations at the macroscopic level including Galilean invariance and a velocity-independent pressure. However, there is a trade-off. The lattice Boltzmann method no longer has the 
pure Boolean operation and numerical stability guaranteed by LGA.

Detailed study of the cavity flow problem using the lattice Boltzmann method has shown that the method is accurate compared with conventional methods using the same mesh size. This verification produces confidence to apply the method to other complex systems. All aspects of the present work such as boundary conditions, parameter ranges, lattice size and compressibility effects are important when the method is applied to other problems. The following remarks are in order:

1. The proper implementation of the boundary conditions is crucial for the lattice Boltzmann simulation. Various boundary conditions such as periodic, particle bounceback, wind tunnel and constant flux conditions are commonly used for different situations in LBE. It is important that the boundary conditions applied for the simulation represent the correct physical problem. In the cavity simulation, for example, besides the uniform top velocity and no-slip conditions on the wall, the mass must be conserved globally. Any violation of this restriction will produce nonphysical results. Be aware that some improper boundary conditions can give a qualitatively reasonable flow but lead to quantitatively incorrect results.

2. The range of parameters for the model is explored for the cavity simulations. Parameters such as the lattice size, maximum velocity, the ratio of rest and moving particle and the single relaxation time are adjustable in LBE. The lattice size should be chosen so that a good resolution for all scales in the problem can be obtained at an affordable cost. The maximum velocity used in a simulation should be properly small for a low Mach number and hence low compressibility requirement and for the validation of the equilibrium distribution which is an expansion of small velocity. For the Chapman-Enskog expansion to be 
valid, the spatial gradients of density and velocity should be small also. Since the maximum velocity and lattice size are limited, the single relaxation time needs to be small to achieve the higher Reynolds numbers. It is found that the lowest relaxation time leading to stable simulations depends on the ratio of rest and moving particles, the maximum velocity and the problem. To obtain a reliable simulation, the relaxation time should be chosen not too close to the lowest limit for the problem under investigation. On the other hand, since the lattice spacing in the LBE is unit, the parameter, $\tau$, can be understood as a mean free path. Therefore, $\tau$ should be small enough compared with macroscopic characteristic length scale. This is a necessary condition that the microscopic statistics of the LBE will approach the Navier-Stokes equations as shown in the multiscale expansion (see the Appendix).

3. The compressibility effect may become important when physical quantities of the smallest scale in an incompressible flow is comparable to the compressibility error. Using a smaller maximum velocity can reduce this error. However, it is probably not practical to predict small scales on the order of $10^{-8}$ or smaller by the present LBE method.

4. The square lattice is better than the triangular lattice (FHP) in two-dimensional simulations because the former can reach higher values of Re number for the same lattice size and maximum velocity.

5. The computer time used in the simulations is not compared carefully with other methods since the LBE includes transient effects in this problem and hence is not economical compared with the multigrid method. There is no doubt, however, that the method can simulate unsteady and other complex problems on a parallel computer with time comparable, if not superior, to other methods.

Lattice gas and lattice Boltzmann methods are relatively new approaches for transport 
phenomena. It is apparent that further research on both theoretical and practical aspects is needed. Implementation of higher order boundary conditions, models for better resolving the small scale phenomena, applications in new fields and to discover new physics, improvement of thermodynamical models and careful studies for three-dimensional geometries are challenges for future research.

\section{Acknowledgments}

Discussions with Drs. Li-shi Luo, Daryl Grunau, Xiaowen Shan, Jim Sterling, Yue Hong Qian and Norman Zabusky are appreciated. This work is supported by the Department of Energy at Los Alamos National Laboratory. The authors wish to acknowledge the Advanced Computing Laboratory of Los Almos National Laboratory for providing us use of the connection machines, CM-200 and CM-5, located at this facility. Hou and Cogley from Kansas State University gratefully appreciate support from IBM, the National Science Foundation (Grant DDM-9113780) and the Kansas Space Grant Consortium. 


\section{Reference}

1. U. Frisch, B. Hasslacher and Y. Pomeau, Phys. Rev. Lett. 56, 1505 (1986).

2. U. Frisch, D. d'Humières, B. Hasslacher P. Lallemand, Y. Pomeau and J-P Rivet, Complex Systems 1, 649 (1987).

3. S. Wolfram, J. Stat. Phys. 45, 471 (1986).

4. Lattice Gas Methods for Partial Differential Equations, edited by G.D. Doolen (AddisonWesley Publishing Company, 1989).

5. Lattice Gas Methods: Theory, Applications and Hardware, edited by G.D. Doolen (Phys-

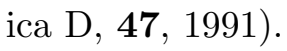

6. G. McNamara and G. Zanetti, Phys. Rev. Lett. 61, 2332 (1988).

7. F. Higuera and J. Jimenez, Europhys. Lett. 9, 663 (1989).

8. F. Higuera and S. Succi, Europhys. Lett. 8, 517 (1989).

9. S. Chen, H. Chen, D. Martinez and W.H. Mattaeus, Phys. Rev. Lett. 67, 3776 (1991).

10. Y. Qian, D. d'Humières and P. Lallemand, Europhys. Lett. 17 (6), 479 (1992).

11. H. Chen, S. Chen and W.H. Matthaeus, Phys. Rev. A 45, 5339 (1992).

12. P.L. Bhatnagar, E.P. Gross and M. Krook, Phys. Rev. 94, 511 (1954).

13. S. Succi, R. Benzi and F. Higuera, Physica D 47, 219, (1991).

14. S. Chen, Z. Wang, X. Shan and G.D. Doolen, J. Stat. Phys. 68, 379 (1992). 
15. G. McNamara and B. Alder, "Lattice Boltzmann simulation of high Reynolds number fluid flow in two dimensions" Microscopic Simulations of Complex Hydrodynamic Phenomena, edited by M. Mareschal and B.L. Holien, (Plenum, New York, 1992).

16. D.O. Mattinez W.H. Matthaeus, S. Chen and D.C. Montgomery, "Comparison of spectral Method and lattice Boltzmann simulations of two-dimensional hydrodynamics" Phys. Fluids $A$, (in press).

17. D. Rothman, J. Geophys. Res. 95, 8663 (1990).

18. S. Chen, K. Diemer, G.D. Doolen, K. Eggert, C. Fu, S. Gutman and B.J. Travis, Physica D 47, 72, (1991).

19. H. Chen and W.H. Matthaeus, Phys. Rev. Lett. 58, 1845 (1987).

20. D. Martinez, S Chen and W.H. Matthaeus, "Lattice Boltzmann Magnetohydrodynamics", submitted to Physics of Plasma, (1993).

21. D. Rothman and J. M. Keller, J. Stat. Phys. 52, 1119 (1988).

22. J.A. Somers and P.. Rem, Physica D 47, 39 (1991).

23. D. Grunau, S. Chen and K. Eggert Phys. Fluids A 5 (10), 2557 (1993).

24. X. Shan and H. Chen, Phys. Rev. E 47 (3), 1815 (1993).

25. D. Dab, A. Lawniczak, J.-P. Boon and R. Kapral, Phys. Rev. Lett. 64, 2462 (1990).

26. R. Kapral, A. Lawniczak and P. Masiar, Phys. Rev. Lett. 66, 2539 (1991).

27. S. Ponce Dawson, S. Chen, and G. Doolen, J. Chem. Phys. 98, 1514 (1993). 
28. Discrete Kinetic Theory, Lattice Gas Dynamics and Foundations of Hydrodynamics, edited by R. Monaco (World Scientific, 1988).

29. J. Stat. Phys., 68 No. 3/4, 1992, edited by J.P. Boon.

30. G.K. Batchelor, Introduction to fluid dynamics, 147 (1956).

31. G.K. Batchelor, J. Fluid Mech. 1, 177 (1956).

32. O. Burggraf, J. Fluid Mech. 24, 113 (1966).

33. F. Pan and A. Acrivos, J. Fluid Mech. 28, 643 (1967).

34. J.D. Bozeman and C. Dalton, J. Comput. Phys. 12, 348 (1973).

35. A.S. Benjamin and V.E. Denny, J. Comput. Phys. 33, 340 (1979).

36. U. Ghia, K.N. Ghia, and C.Y. Shin, J. Comput. Phys. 48, 387 (1982).

37. R. Schreiber and H.B. Keller, J. Comput. Phys. 49, 310 (1983).

38. S.P. Vanka, J. Comput. Phys. 65, 138 (1986).

39. M. Zhou, C.L. Huang and Q. Zou, "A Multigrid Method for the High-Re Solutions of Navier-Stokes Equations in Primitive Variables" preprint, (1992).

40. P. Demaret and M.O. Deville, J. Comput. Phys. 95, 359 (1991).

41. P.A. Skordos, "Initial and boundary conditions for the lattice Boltzmann methods" preprint (1993).

42. D.P. Ziegler, J. Stat. Phys. Vol.71, Nos. 5/6, (1993). 
43. K. Atkinson, Elementary Numerical Analysis, 2nd Ed., John Wiley \& Sons, Inc., New York, 173-175 (1993).

44. Q. Zou, S. Hou, S. Chen and G.D. Doolen, in preparation.

45. J.D. Sterling and S. Chen, "Stability analysis of lattice Boltzmann method", submitted to J. Comput. Phys., (1993).

\section{Figure captions}

Figure 1. (a) Stream function for $R e=10$. Top velocity $U=0.1$ The center of the primary vortex is at $(0.5216,0.7686)$. The center of lower left vortex is at $(0.0392,0.0431)$. The center of lower right vortex is at $(0.9647,0.0392)$.

Figure 1. (b) Stream function for $R e=100$. Top velocity $U=0.01$. The center of the primary vortex is at $(0.6196,0.7373)$. The center of lower left vortex is at $(0.0392,0.0353)$. The center of lower right vortex is at $(0.9495,0.0353)$.

Figure 1. (c) Stream function for $R e=400$. Top velocity $U=0.1$. The center of the primary vortex is at $(0.5608,0.6078)$. The center of lower left vortex is at $(0.0549,0.0510)$. The center of lower right vortex is at $(0.8902,0.1255)$.

Figure 1. (d) Stream function for $R e=1000$. Top velocity $U=0.1$. The center of the primary vortex is at $(0.5333,0.5647)$. The center of lower left vortex is at $(0.0902,0.0784)$. The center of lower right vortex is at $(0.8667,0.1137)$.

Figure 1. (e) Stream function for $R e=2000$. Top velocity $U=0.1$. The center of the primary vortex is at $(0.5255,0.5490)$. The center of lower left vortex is at $(0.0902,0.1059)$. The center of lower right vortex is at $(0.8471,0.0980)$.

Figure 1. (f) Stream function for $R e=5000$. Top velocity $U=0.1$. The center of the 
primary vortex is at $(0.5176,0.5373)$. The center of lower left vortex is at $(0.0784,0.1373)$. The center of lower right vortex is at $(0.8078,0.0745)$.

Figure 1. (g) Stream function for $R e=7500$. Top velocity $U=0.1$. The center of the primary vortex is at $(0.5176,0.5333)$. The center of lower left vortex is at $(0.0706,0.1529)$. The center of lower right vortex is at $(0.7922,0.0667)$.

Figure 2. The locations of the center of the primary vortex for different values of Re numbers. The origin is the geometric center of the cavity.

Figure 3. (a) Velocity profiles for $\mathrm{u}$ through the geometric center of the cavity.

Figure 3. (b) Velocity profiles for $\mathrm{v}$ through the geometric center of the cavity.

Figure 4. Vorticity contours of the cavity flow. (a) $R e=10$. (b) $R e=100$. (c) $R e=400$. (d) $R e=1000$. (e) $R e=2000$. (f) $R e=5000$. (g) $R e=7500$.

Figure 5. Pressure deviation contours of the cavity flow. (a) $R e=10$. (b) $R e=100$. (c) $\operatorname{Re}=400$. (d) $R e=1000$. (e) $R e=2000$. (f) $R e=5000$. (g) $R e=7500$.

Figure 6. Drag coefficient of top wall versus. Re.

Figure 7. Convergence rate of the LBE method for cavity flow at Re=1000 with top velocity $\mathrm{U}=0.1$. The errors are calculated relative to results obtained on a $513 \times 513$ lattice. 


\section{Appendix}

This appendix details the derivation that shows how the Navier-Stokes equations are recovered from a lattice Boltzmann equation on a square lattice by using the ChapmanEnskog expansion procedure of kinetic theory. In addition, the equilibrium distribution functions are obtained to guarantee that the requirements of isotropy, Galilean-invariance and velocity-independent pressure are satisfied.

On each node of a square lattice there are three types of particle, namely, a rest particle, a particle moving along perpendicular directions and a moving particle along diagonal directions. (see Figure 1A).

Figure 1A Schematic of a square lattice

The velocity vectors $\mathbf{e}_{1, i}, \mathbf{e}_{2, i}$ are defined as

$$
\begin{gathered}
\mathbf{e}_{1, i}=\left(\cos \frac{i-1}{2} \pi, \sin \frac{i-1}{2} \pi\right) \quad i=1, \cdots, 4 \\
\mathbf{e}_{2, i}=\sqrt{2}\left(\cos \left(\frac{i-1}{2} \pi+\frac{\pi}{4}\right), \sin \left(\frac{i-1}{2} \pi+\frac{\pi}{4}\right)\right) \quad i=1, \cdots, 4
\end{gathered}
$$


The symmetric properties of the tensor

$$
\sum_{i}\left(e_{\sigma i \alpha} e_{\sigma i \beta} \cdots\right)
$$

are needed in the derivation and given as follows:

The odd order of tensors are equal to zero, i.e.

$$
\begin{gathered}
\sum_{i} \mathbf{e}_{\sigma i}=0, \quad \sigma=1,2 \\
\sum_{i} e_{\sigma i \alpha} e_{\sigma i \beta} e_{\sigma i \gamma}=0, \quad \alpha, \beta, \gamma=1,2,
\end{gathered}
$$

and

$$
\sum_{i} e_{\sigma i \alpha} e_{\sigma i \beta} e_{\sigma i \gamma} e_{\sigma i \theta} e_{\sigma i \zeta}=0, \quad \alpha, \beta, \gamma, \theta, \zeta=1,2
$$

The second order of tensor satisfies

$$
\sum_{i} e_{\sigma i \alpha} e_{\sigma i \beta}=2 e_{\sigma}^{2} \delta_{\alpha \beta}, \quad \alpha, \beta=1,2
$$

where

$$
\delta_{\alpha, \beta}=\left\{\begin{array}{cc}
1, & \alpha=\beta \\
0, & \alpha \neq \beta
\end{array}\right.
$$

and

$$
e_{\sigma}= \begin{cases}1, & \sigma=1 \\ \sqrt{2}, & \sigma=2\end{cases}
$$

is the length of $\mathbf{e}_{\sigma i}$.

Finally, the fourth order tensor has an expression as

$$
\sum_{i} e_{\sigma i \alpha} e_{\sigma i \beta} e_{\sigma i \gamma} e_{\sigma i \theta}= \begin{cases}2 \delta_{\alpha \beta \gamma \theta}, & \sigma=1 \\ 4 \Delta_{\alpha \beta \gamma \theta}-8 \delta_{\alpha \beta \gamma \theta}, & \sigma=2\end{cases}
$$


where

$$
\delta_{\alpha \beta \gamma \theta}= \begin{cases}1, & \alpha=\beta=\gamma=\theta, \\ 0, & \text { otherwise }\end{cases}
$$

and

$$
\Delta_{\alpha \beta \gamma \theta}=\left(\delta_{\alpha \beta} \delta_{\gamma \theta}+\delta_{\alpha \gamma} \delta_{\beta \theta}+\delta_{\alpha \theta} \delta_{\beta \gamma}\right)
$$

Here the subscripts, $\sigma$, denote the types of particle; $i$ indicates the directions of particle movement and $\alpha, \beta, \gamma, \theta$, and $\zeta$ are the components of the coordinates.

The Chapman-Enskog procedure is an asymptotic expansion method for solving the Boltzmann equation in kinetic theory. It is necessary to introduce a small parameter in an asymptotic expansion to compare orders. The lattice constants are required to be small compared with macroscopic characteristic length scale, for example, the edge length of the cavity, $L$, i.e. $\left|\mathbf{e}_{\sigma i}\right| / L \ll 1$. Using $\epsilon$ as a measure of this small scale, instead of using a unit lattice constant and time step as in Section 2, the lattice Boltzmann equation is written as

$$
f_{\sigma i}\left(\mathbf{x}+\epsilon \mathbf{e}_{\sigma i}, t+\epsilon\right)-f_{\sigma i}(\mathbf{x}, t)=\Omega_{\sigma i}
$$

where $\Omega_{\sigma i}$ is the collision operator and $\epsilon$ is solely used for distinguishing different orders. The lattice Boltzmann BGK equation is

$$
f_{\sigma i}\left(\mathbf{x}+\epsilon \mathbf{e}_{\sigma i}, t+\epsilon\right)-f_{\sigma i}(\mathbf{x}, t)=-\frac{1}{\tau}\left[f_{\sigma i}(\mathbf{x}, t)-f_{\sigma i}^{(0)}(\mathbf{x}, t)\right]
$$

where $f_{\sigma i}(\sigma=0 \quad i=1 ; \quad \sigma=1,2 \quad i=1, \cdots, 4)$ is the single-particle distribution function, $f_{\sigma i}^{(0)}(\mathbf{x}, t)$ is the equilibrium distribution at $\mathbf{x}, t$ and $\tau$ is the single relaxation time. A general form of $f_{\sigma i}^{(0)}(\mathbf{x}, t)$ can be taken as

$$
f_{\sigma i}^{(0)}(\mathbf{x}, t)=A_{\sigma}+B_{\sigma}\left(\mathbf{e}_{\sigma i} \cdot \mathbf{u}\right)+C_{\sigma}\left(\mathbf{e}_{\sigma i} \cdot \mathbf{u}\right)^{2}+D_{\sigma} u^{2} .
$$


Here $A_{\sigma}, B_{\sigma}, C_{\sigma}$ and $D_{\sigma}$ are cofficients that need to be found and depend on $\rho$, but not on $u$. Equation (8) can be thought as a special type of small velocity (up to the $u^{2}$ term) expansion of $f_{\sigma i}^{(0)}$.

It is obvious that $B_{0}=C_{0}=0$ and Eq. (8) can be written separately in the form

$$
\begin{array}{lr}
f_{01}^{(0)}(\mathbf{x}, t)=A_{0} & +D_{0} u^{2}, \\
f_{1 i}^{(0)}(\mathbf{x}, t) & =A_{1}+B_{1}\left(\mathbf{e}_{1 i} \cdot \mathbf{u}\right)+C_{1}\left(\mathbf{e}_{1 i} \cdot \mathbf{u}\right)^{2}+D_{1} u^{2}, \\
f_{2 i}^{(0)}(\mathbf{x}, t) & =A_{2}+B_{1}\left(\mathbf{e}_{2 i} \cdot \mathbf{u}\right)+C_{2}\left(\mathbf{e}_{2 i} \cdot \mathbf{u}\right)^{2}+D_{2} u^{2},
\end{array}
$$

where $f_{\sigma i}^{(0)}$ and $f_{\sigma i}$ satisfy the following constraints:

$$
\sum_{\sigma} \sum_{i} f_{\sigma i}=\sum_{\sigma} \sum_{i} f_{\sigma i}^{(0)}=\rho
$$

and

$$
\sum_{\sigma} \sum_{i} f_{\sigma i} \mathbf{e}_{\sigma i}=\sum_{\sigma} \sum_{i} f_{\sigma i}^{(0)} \mathbf{e}_{\sigma i}=\rho \mathbf{u} .
$$

These constraints mean that the non-equilibrium distributions do not contribute to the local values of density and momentum. Using Eqs. (10), (11), (11) and (1), some constraints for coefficients $A_{\sigma}, B_{\sigma}, C_{\sigma}$ and $D_{\sigma}$ are found to be

$$
\begin{gathered}
A_{0}+4 A_{1}+4 A_{2}=\rho \\
2 C_{1}+4 C_{2}+D_{0}+4 D_{1}+4 D_{2}=0
\end{gathered}
$$

and

$$
2 B_{1}+4 B_{2}=\rho
$$

Starting from the LBE with BGK collision operator, the Navier-Stokes equations can be recovered. Taking a Taylor expansion of Eq. (7) gives

$$
f_{\sigma i}\left(\mathbf{x}+\epsilon \mathbf{e}_{\sigma i}, t+\epsilon\right)-f_{\sigma i}(\mathbf{x}, t)=\sum_{n=0}^{\infty} \frac{\epsilon^{n}}{n !}\left[\frac{\partial}{\partial t}+\left(\mathbf{e}_{\sigma i} \cdot \nabla\right)\right]^{n} f_{\sigma i}(\mathbf{x}, t),
$$


where

$$
\mathbf{e}_{\sigma i} \cdot \nabla=e_{\sigma i \alpha} \partial_{\alpha}+e_{\sigma i \beta} \partial_{\beta}
$$

and the Einstein summation convention is used. If terms up to $O\left(\epsilon^{2}\right)$ are retained in Eq (15), the results is

$$
\begin{aligned}
\epsilon\left[\frac{\partial}{\partial t}+\left(\mathbf{e}_{\sigma i} \cdot \nabla\right)\right] f_{\sigma i}+\frac{\epsilon^{2}}{2} & {\left[\frac{\partial}{\partial t}+\left(\mathbf{e}_{\sigma i} \cdot \nabla\right)\right]^{2} f_{\sigma i}+O\left(\epsilon^{3}\right) } \\
& =-\frac{1}{\tau}\left[f_{\sigma i}(\mathbf{x}, t)-f_{\sigma i}^{(0)}(\mathbf{x}, t)\right] .
\end{aligned}
$$

Next, the Chapman-Enskog-like expansion is applied to Eq.(16). Expanding $f_{\sigma i}$ about $f_{\sigma i}^{(0)}$ gives

$$
f_{\sigma i}=\sum_{n=0}^{\infty} \epsilon^{n} f_{\sigma i}^{(n)}=f_{\sigma i}^{(0)}+\epsilon f_{\sigma i}^{(1)}+\epsilon^{2} f_{\sigma i}^{(2)}+\cdots,
$$

with constraints

$$
\sum_{\sigma} \sum_{i} f_{\sigma i}^{(0)}\left[\begin{array}{c}
1 \\
\mathbf{e}_{\sigma i}
\end{array}\right]=\left[\begin{array}{c}
\rho \\
\rho \mathbf{u}
\end{array}\right]
$$

and

$$
\sum_{\sigma} \sum_{i} f_{\sigma i}^{(n)}\left[\begin{array}{c}
1 \\
\mathbf{e}_{\sigma i}
\end{array}\right]=0, \quad n \geq 1 .
$$

The collision operator, $\Omega_{\sigma i}$, becomes

$$
-\frac{1}{\tau}\left[\epsilon f_{\sigma i}^{(1)}+\epsilon^{2} f_{\sigma i}^{(2)}+\cdots\right]
$$

To discuss changes in different time scales, three time scales, $t_{0}, t_{1}, t_{2}$ are introduced as

$$
t_{0}=t, \quad t_{1}=\epsilon t, \quad t_{2}=\epsilon^{2} t
$$

where

$$
\frac{\partial}{\partial t}=\frac{\partial}{\partial t_{0}}+\epsilon \frac{\partial}{\partial t_{1}}+\epsilon^{2} \frac{\partial}{\partial t_{2}} .
$$


Substituting Eqs. (17), (20), and (21) into Eq (16), the equation to order of $\epsilon$ is:

$$
\left(\partial_{t_{0}}+\mathbf{e}_{\sigma i} \cdot \nabla\right) f_{\sigma i}^{(0)}=-\frac{1}{\tau} f_{\sigma i}^{(1)}
$$

The equation to order of $\epsilon^{2}$ is:

$$
\partial_{t_{0}} f_{\sigma i}^{(1)}+\partial_{t_{1}} f_{\sigma i}^{(0)}+\left(\mathbf{e}_{\sigma i} \cdot \nabla\right) f_{\sigma i}^{(1)}+\frac{1}{2}\left[\frac{\partial}{\partial t_{0}}+\left(\mathbf{e}_{\sigma i} \cdot \nabla\right)\right]^{2} f_{\sigma i}^{(0)}=-\frac{1}{\tau} f_{\sigma i}^{(2)}
$$

Using Eq. (22), one obtains

$$
\left(\partial_{t_{0}}+\mathbf{e}_{\sigma i} \cdot \nabla\right)^{2} f_{\sigma i}^{(0)}=-\frac{1}{\tau}\left(\partial_{t_{0}}+\mathbf{e}_{\sigma i} \cdot \nabla\right) f_{\sigma i}^{(1)}
$$

Substituting Eq. (24) into Eq. (23) leads to

$$
\partial_{t_{1}} f_{\sigma i}^{(0)}+\left(1-\frac{1}{2 \tau}\right)\left(\partial_{t_{0}}+\mathbf{e}_{\sigma i} \cdot \nabla\right) f_{\sigma i}^{(1)}=-\frac{1}{\tau} f_{\sigma i}^{(2)}
$$

To derive the equations for $\rho$ and $\rho \mathbf{u}$ to the first order in $\epsilon$, a summation of Eq. (22) with respect to $\sigma$ and $i$ is taken to give

$$
\partial_{t_{0}} \sum_{\sigma} \sum_{i} f_{\sigma i}^{(0)}+\sum_{\sigma} \sum_{i}\left(\mathbf{e}_{\sigma i} \cdot \nabla\right) f_{\sigma i}^{(0)}=-\frac{1}{\tau} \sum_{\sigma} \sum_{i} f_{\sigma i}^{(1)}=0
$$

which is the first order continuity equation

$$
\partial_{t_{0}} \rho+\nabla \cdot(\rho \mathbf{u})=0
$$

Similarly, multiplying $\mathbf{e}_{\sigma i}$ in Eq. (22) and taking summation as above gives

$$
\partial_{t_{0}} \sum_{\sigma} \sum_{i} f_{\sigma i}^{(0)} \mathbf{e}_{\sigma i}+\sum_{\sigma} \sum_{i}\left(\mathbf{e}_{\sigma i} \cdot \nabla\right) f_{\sigma i}^{(0)} \mathbf{e}_{\sigma i}=-\frac{1}{\tau} \sum_{\sigma} \sum_{i} f_{\sigma i}^{(1)} \mathbf{e}_{\sigma i}=0
$$

which can be simplified to

$$
\partial_{t_{0}}(\rho \mathbf{u})+\nabla \cdot \sum_{\sigma} \sum_{i}\left(\mathbf{e}_{\sigma i} \mathbf{e}_{\sigma i}\right) f_{\sigma i}^{(0)}=0 .
$$


Defining the momentum flux tensor as

$$
\Pi=\sum_{\sigma} \sum_{i}\left(\mathbf{e}_{\sigma i} \mathbf{e}_{\sigma i}\right) f_{\sigma i}
$$

Eq. (27) can be rewritten as

$$
\partial_{t_{0}}(\rho \mathbf{u})+\nabla \cdot \Pi^{(0)}=0
$$

Similarly, the equations of order of $\epsilon^{2}$ for $\rho$ and $\mathbf{u}$ can be obtained from Eq. (25) as

$$
\partial_{t_{1}} \rho+\left(1-\frac{1}{2 \tau}\right) \partial_{t_{0}} \sum_{\sigma} \sum_{i} f_{\sigma i}^{(1)}+\left(1-\frac{1}{2 \tau}\right) \nabla \cdot \sum_{\sigma} \sum_{i} f_{\sigma i}^{(1)} \mathbf{e}_{\sigma i}=-\frac{1}{\tau} \sum_{\sigma} \sum_{i} f_{\sigma i}^{(2)}=0
$$

and

$\partial_{t_{1}}(\rho \mathbf{u})+\left(1-\frac{1}{2 \tau}\right) \partial_{t_{0}} \sum_{\sigma} \sum_{i} f_{\sigma i}^{(1)} \mathbf{e}_{\sigma i}+\left(1-\frac{1}{2 \tau}\right) \nabla \cdot \sum_{\sigma} \sum_{i} \mathbf{e}_{\sigma i} \mathbf{e}_{\sigma i} f_{\sigma i}^{(1)}=-\frac{1}{\tau} \sum_{\sigma} \sum_{i} f_{\sigma i}^{(2)} \mathbf{e}_{\sigma i}=0$

Here the constraint given by Eq. (18) is used. By applying another constraint, Eq. (19), these two equations can be simplified to

$$
\partial_{t_{1}} \rho=0
$$

and

$$
\partial_{t_{1}}(\rho \mathbf{u})+\left(1-\frac{1}{2 \tau}\right) \nabla \cdot \Pi^{(1)}=0 .
$$

Write $\Pi^{(0)}$ as follows:

$$
\begin{aligned}
& \Pi_{\alpha \beta}^{(0)}=\sum_{\sigma} \sum_{i} e_{\sigma i \alpha} e_{\sigma i \beta} f_{\sigma i}^{(0)} \\
& =\sum_{\sigma} \sum_{i} e_{\sigma i \alpha} e_{\sigma i \beta}\left(A_{\sigma}+B_{\sigma} e_{\sigma i \gamma} u_{\gamma}+C_{\sigma} e_{\sigma i \gamma} e_{\sigma i \theta} u_{\gamma} u_{\theta}+D_{\sigma} u^{2}\right) \\
& =\sum_{\sigma} A_{\sigma} 2 e_{\sigma}^{2} \delta_{\alpha \beta}+\sum_{\sigma} C_{\sigma} u_{\gamma} u_{\theta} \sum_{i} e_{\sigma i \alpha} e_{\sigma i \beta} e_{\sigma i \gamma} e_{\sigma i \theta}+\sum_{\sigma} D_{\sigma} 2 e_{\sigma}^{2} \delta_{\alpha \beta} u^{2} \\
& =\left(2 A_{1}+4 A_{2}\right) \delta_{\alpha \beta}+C_{1} u_{\gamma} u_{\theta}\left(2 \delta_{\alpha \beta \gamma \theta}\right) \\
& +C_{2} u_{\gamma} u_{\theta}\left(4 \Delta_{\alpha \beta \gamma \theta}-8 \delta_{\alpha \beta \gamma \theta}\right)+\left(2 D_{1} u^{2}+4 D_{2} u^{2}\right) \delta_{\alpha \beta} .
\end{aligned}
$$


Since

$$
u_{\gamma} u_{\theta} \delta_{\alpha \beta \gamma \theta}=u_{\alpha} u_{\beta} \delta_{\alpha \beta}
$$

and

$$
4 u_{\gamma} u_{\theta} \Delta_{\alpha \beta \gamma \theta}=4\left(\delta_{\alpha \beta} \delta_{\gamma \theta}+\delta_{\alpha \gamma} \delta_{\beta \theta}+\delta_{\alpha \theta} \delta_{\beta \gamma}\right) u_{\gamma} u_{\theta}=4 \delta_{\alpha \beta} u^{2}+8 u_{\alpha} u_{\beta}
$$

one can write $\Pi_{\alpha \beta}^{(0)}$ as

$$
\Pi_{\alpha \beta}^{(0)}=\left[2 A_{1}+4 A_{2}+\left(4 C_{2}+2 D_{1}+4 D_{2}\right) u^{2}\right] \delta_{\alpha \beta}+8 C_{2} u_{\alpha} u_{\beta}+\left(2 C_{1}-8 C_{2}\right) u_{\alpha} u_{\beta} \delta_{\alpha \beta} .
$$

The first term is the pressure term and the other two are nonlinear terms. In order to obtain velocity-independent pressure, the coefficient of $u^{2}$ is chosen to satisfy

$$
4 C_{2}+2 D_{1}+4 D_{2}=0
$$

To have Galilean invariance, the non-isotropic term is eliminated by choosing

$$
2 C_{1}-8 C_{2}=0
$$

Eq. (32) becomes

$$
\Pi_{\alpha \beta}^{(0)}=\left(2 A_{1}+4 A_{2}\right) \delta_{\alpha \beta}+8 C_{2} u_{\alpha} u_{\beta}
$$

Assuming that

$$
8 C_{2}=\rho
$$

and

$$
2 A_{1}+4 A_{2}=c_{s}^{2} \rho
$$

where $c_{s}$ is speed of sound, gives the final expression for $\Pi^{(0)}$ as

$$
\Pi_{\alpha \beta}^{(0)}=c_{s}^{2} \rho \delta_{\alpha \beta}+\rho u_{\alpha} u_{\beta} .
$$


Substituting Eq. (38) into Eq. (29) results in

$$
\frac{\partial(\rho \mathbf{u})}{\partial t_{0}}+\nabla \cdot(\rho \mathbf{u u})=-\nabla\left(c_{s}^{2} \rho\right)
$$

Eqs. (26) and (39) are Euler equations that are derived from the $\epsilon$-order of the expansion of the Boltzmann equation.

To derive the equations accurate to $\epsilon^{2}$, the quantity $\nabla \cdot \Pi^{(1)}$ needs to be evaluated. From Eq. (22) the non-equilibrium distribution can be expressed as

$$
f_{\sigma i}^{(1)}=-\tau \partial_{t_{0}} f_{\sigma i}^{(0)}-\tau\left(e_{\sigma i \gamma} \partial_{\gamma}\right) f_{\sigma i}^{(0)}
$$

Substituting $f_{\sigma i}^{(1)}$ into $\Pi_{\alpha \beta}^{(1)}$ gives

$$
\begin{aligned}
& \Pi_{\alpha \beta}^{(1)}=\sum_{\sigma} \sum_{i} e_{\sigma i \alpha} e_{\sigma i \beta} f_{\sigma i}^{(1)}=-\tau \partial_{t_{0}} \sum_{\sigma} \sum_{i} e_{\sigma i \alpha} e_{\sigma i \beta} f_{\sigma i}^{(0)}-\tau \partial_{\gamma} \sum_{\sigma} \sum_{i} e_{\sigma i \alpha} e_{\sigma i \beta} e_{\sigma i \gamma} f_{\sigma i}^{(0)} \\
& =-\tau\left\{\partial_{t_{0}} \Pi_{\alpha \beta}^{(0)}+\partial_{\gamma} \sum_{\sigma} \sum_{i} e_{\sigma i \alpha} e_{\sigma i \beta} e_{\sigma i \gamma}\left(A_{\sigma}+B_{\sigma} e_{\sigma i \theta} u_{\theta}+C_{\sigma} e_{\sigma i \theta} e_{\sigma i s} u_{\theta} u_{s}+D_{\sigma} u^{2}\right)\right\}
\end{aligned}
$$

Using Eq. (38) for $\Pi_{\alpha \beta}^{(0)}$ leads to

$$
\begin{aligned}
\Pi_{\alpha \beta}^{(1)} & =-\tau\left\{\partial_{t_{0}}\left[\left(c_{s}^{2} \rho\right) \delta_{\alpha \beta}+\rho u_{\alpha} u_{\beta}\right]+\partial_{\gamma} B_{1} u_{\theta} 2 \delta_{\alpha \beta \gamma \theta}+\partial_{\gamma} B_{2} u_{\theta}\left(4 \Delta_{\alpha \beta \gamma \theta}-8 \delta_{\alpha \beta \gamma \theta}\right)\right\} \\
& =-\tau\left\{-c_{s}^{2} \delta_{\alpha \beta} \partial_{\gamma}\left(\rho u_{\gamma}\right)+\partial_{t_{0}}\left(\rho u_{\alpha} u_{\beta}\right)+\partial_{\alpha}\left(2 B_{1}-8 B_{2}\right) u_{\beta} \delta_{\alpha \beta}\right. \\
& \left.+4 \partial_{\gamma}\left(B_{2} u_{\gamma}\right) \delta_{\alpha \beta}+4 \partial_{\alpha}\left(B_{2} u_{\beta}\right)+4 \partial_{\beta}\left(B_{2} u_{\alpha}\right)\right\}
\end{aligned}
$$

To avoid non-isotropy, set

$$
2 B_{1}-8 B_{2}=0
$$

Recalling Eq. (14), $B_{1}$ and $B_{2}$ can be uniquely determined as

$$
B_{2}=\frac{\rho}{12}, \quad B_{1}=\frac{\rho}{3}
$$

Therefore, Eq. (41) can be written as

$$
\Pi_{\alpha \beta}^{(1)}=-\tau\left\{\frac{1}{3} \partial_{\gamma}\left(\rho u_{\gamma}\right) \delta_{\alpha \beta}+\frac{1}{3} \partial_{\alpha}\left(\rho u_{\beta}\right)+\frac{1}{3} \partial_{\beta}\left(\rho u_{\alpha}\right)-c_{s}^{2} \partial_{\gamma}\left(\rho u_{\gamma}\right) \delta_{\alpha \beta}+\partial_{t_{0}}\left(\rho u_{\alpha} u_{\beta}\right)\right\}
$$


The last term can be simplified using Eq. (39) to take the form

$$
\begin{aligned}
& \partial_{t_{0}}\left(\rho u_{\alpha} u_{\beta}\right)=u_{\beta} \partial_{t_{0}}\left(\rho u_{\alpha}\right)+\rho u_{\alpha} \partial_{t_{0}} u_{\beta} \\
= & u_{\beta}\left[-\partial_{\gamma}\left(\rho u_{\alpha} u_{\gamma}\right)-\partial_{\alpha}\left(c_{s}^{2} \rho\right)\right]+u_{\alpha} \partial_{t_{0}}\left(\rho u_{\beta}\right)-u_{\alpha} u_{\beta} \partial_{t_{0}} \rho \\
= & -u_{\beta} \partial_{\gamma}\left(\rho u_{\alpha} u_{\gamma}\right)-u_{\beta} \partial_{\alpha}\left(c_{s}^{2} \rho\right)-u_{\alpha}\left[\partial_{\gamma}\left(\rho u_{\beta} u_{\gamma}\right)+\partial_{\beta}\left(c_{s}^{2} \rho\right)\right]+u_{\alpha} u_{\beta} \partial_{\gamma}\left(\rho u_{\gamma}\right) \\
= & -u_{\alpha} \partial_{\beta}\left(c_{s}^{2} \rho\right)-u_{\beta} \partial_{\alpha}\left(c_{s}^{2} \rho\right)-u_{\alpha} u_{\beta} \partial_{\gamma}\left(\rho u_{\gamma}\right)-\rho u_{\alpha} u_{\gamma} \partial_{\gamma} u_{\beta}+u_{\alpha} u_{\beta} \partial_{\gamma}\left(\rho u_{\gamma}\right)-u_{\beta} \partial_{\gamma}\left(\rho u_{\alpha} u_{\gamma}\right) \\
= & -u_{\alpha} \partial_{\beta}\left(c_{s}^{2} \rho\right)-u_{\beta} \partial_{\alpha}\left(c_{s}^{2} \rho\right)-\rho u_{\alpha} u_{\gamma} \partial_{\gamma} u_{\beta}-u_{\beta} \partial_{\gamma}\left(\rho u_{\alpha} u_{\gamma}\right) .
\end{aligned}
$$

Eq. (44) therefore becomes

$$
\begin{aligned}
\Pi_{\alpha \beta}^{(1)}=- & \tau\left\{\frac{1}{3} \partial_{\gamma}\left(\rho u_{\gamma}\right) \delta_{\alpha \beta}+\frac{1}{3} \partial_{\alpha}\left(\rho u_{\beta}\right)+\frac{1}{3} \partial_{\beta}\left(\rho u_{\alpha}\right)-c_{s}^{2} \partial_{\gamma}\left(\rho u_{\gamma}\right) \delta_{\alpha \beta}\right. \\
& \left.-u_{\alpha} \partial_{\beta}\left(c_{s}^{2} \rho\right)-u_{\beta} \partial_{\alpha}\left(c_{s}^{2} \rho\right)-\rho u_{\alpha} u_{\gamma} \partial_{\gamma} u_{\beta}-u_{\beta} \partial_{\gamma}\left(\rho u_{\alpha} u_{\gamma}\right)\right\}
\end{aligned}
$$

Note that $\Pi_{\alpha \beta}^{(1)}$ does not only depend on the first spatial derivatives of $\rho$ and $\mathbf{u}$. Ignoring the last two terms which are order of $O\left(u^{3}\right)$ in Eq. (45), leads to

$$
\begin{array}{r}
\Pi_{\alpha \beta}^{(1)}=-\tau\left\{\left(\frac{1}{3}-c_{s}^{2}\right) \partial_{\gamma}\left(\rho u_{\gamma}\right) \delta_{\alpha \beta}+\frac{1}{3} \partial_{\alpha}\left(\rho u_{\beta}\right)\right. \\
\left.+\frac{1}{3} \partial_{\beta}\left(\rho u_{\alpha}\right)-u_{\alpha} \partial_{\beta}\left(c_{s}^{2} \rho\right)-u_{\beta} \partial_{\alpha}\left(c_{s}^{2} \rho\right)\right\}+O\left(u^{3}\right) .
\end{array}
$$

Combine equations of $O(\epsilon)$ and $O\left(\epsilon^{2}\right)$ for $\rho$ and $\mathbf{u}$, and Eqs. (26), (39), (30) and (31) with Eq. (46) as follows:

Eq. (26) added to Eq. (30) multipled by $\epsilon$ gives

$$
\partial_{t_{0}} \rho+\epsilon \partial_{t_{1}} \rho+\nabla \cdot(\rho \mathbf{u})=0
$$

which gives the correct form of the continuity equation as

$$
\frac{\partial \rho}{\partial t}+\nabla \cdot(\rho \mathbf{u})=0
$$


Eq. (39) added to Eq. (31) multipled by $\epsilon$ gives

$$
\partial_{t}(\rho \mathbf{u})+\nabla \cdot(\rho \mathbf{u u})=-\nabla \cdot\left(c_{s}^{2} \rho\right)-\epsilon\left(1-\frac{1}{2 \tau}\right) \nabla \cdot \Pi_{\alpha \beta}^{(1)}
$$

Substituting Eq. (46) for $\Pi_{\beta \alpha}^{(1)}$, Eq. (48) becomes

$$
\begin{gathered}
\partial_{t}\left(\rho u_{\alpha}\right)+\partial_{\beta}\left(\rho u_{\alpha} u_{\beta}\right)=-\partial_{\alpha}\left(c_{s}^{2} \rho\right)+\epsilon\left(\tau-\frac{1}{2}\right) \partial_{\beta}\left\{\left(\frac{1}{3}-c_{s}^{2}\right) \partial_{\gamma}\left(\rho u_{\gamma}\right) \delta_{\alpha \beta}\right. \\
\left.+\frac{1}{3} \partial_{\alpha}\left(\rho u_{\beta}\right)+\frac{1}{3} \partial_{\beta}\left(\rho u_{\alpha}\right)-u_{\alpha} \partial_{\beta}\left(c_{s}^{2} \rho\right)-u_{\beta} \partial_{\alpha}\left(c_{s}^{2} \rho\right)\right\}+O\left(u^{3}\right)+O\left(\epsilon^{3}\right)
\end{gathered}
$$

which may be written in the form

$$
\begin{array}{r}
\partial_{t}\left(\rho u_{\alpha}\right)+\partial_{\beta}\left(\rho u_{\alpha} u_{\beta}\right)=-\partial_{\alpha}\left(c_{s}^{2} \rho\right)+\epsilon\left(\tau-\frac{1}{2}\right)\left\{\partial_{\alpha}\left[\left(\frac{1}{3}-c_{s}^{2}\right) \partial_{\gamma}\left(\rho u_{\gamma}\right)\right]\right. \\
\left.+\partial_{\beta}\left[\frac{1}{3} \rho\left(\partial_{\alpha} u_{\beta}+\partial_{\beta} u_{\alpha}\right)\right]+\partial_{\beta}\left[\left(\frac{1}{3}-c_{s}^{2}\right)\left(u_{\alpha} \partial_{\beta} \rho+u_{\beta} \partial_{\alpha} \rho\right)\right]\right\}+O\left(u^{3}\right)+O\left(\epsilon^{3}\right)
\end{array}
$$

Consider the constraints on $A_{\sigma}$ given in Eqs. (12) and (37), and choosing

$$
A_{0}=\frac{4}{9} \rho, \quad A_{1}=\frac{1}{9} \rho, \quad A_{2}=\frac{1}{36} \rho,
$$

Eq. (12) is satisfied and the sound speed is determined by

$$
c_{s}^{2}=\frac{1}{3}
$$

Eq. (51) is simplified as

$$
\partial_{t}\left(\rho u_{\alpha}\right)+\partial_{\beta}\left(\rho u_{\alpha} u_{\beta}\right)=-\partial_{\alpha}\left(c_{s}^{2} \rho\right)+\frac{1}{3} \epsilon\left(\tau-\frac{1}{2}\right) \partial_{\beta}\left[\rho\left(\partial_{\alpha} u_{\beta}+\partial_{\beta}\left(u_{\alpha}\right)\right]+O\left(u^{3}\right)+O\left(\epsilon^{3}\right)\right.
$$

Define the stain-rate tensor as

$$
S_{\alpha \beta}=\frac{1}{2}\left(\partial_{\alpha} u_{\beta}+\partial_{\beta} u_{\alpha}\right)
$$

Then Eq. (52) can be rewritten as follows:

$$
\partial_{t}\left(\rho u_{\alpha}\right)+\partial_{\beta}\left(\rho u_{\alpha} u_{\beta}\right)=-\partial_{\alpha}\left(c_{s}^{2} \rho\right)+2 \nu \partial_{\beta}\left(\rho S_{\alpha \beta}\right)+O\left(u^{3}\right)+O\left(\epsilon^{3}\right),
$$


where

$$
\nu=\frac{2 \tau-1}{6} \epsilon,
$$

with $\nu$ being the the kinematic viscosity. Recall the Navier-Stokes equations in the twodimensional space $[18]$

$$
\partial_{t}\left(\rho u_{\alpha}\right)+\partial_{\beta}\left(\rho u_{\alpha} u_{\beta}\right)=-\partial_{\alpha} p+\partial_{\beta}\left\{2 \mu\left(S_{\alpha \beta}-\frac{1}{2} u_{\gamma \gamma} \delta_{\alpha \beta}\right)\right\}
$$

and

$$
\partial_{t} \rho+\nabla \cdot(\rho \mathbf{u})=0
$$

For an incompressible fluid with constant viscosity, $\mu$, the Navier-Stokes equations become

$$
\partial_{t}\left(\rho u_{\alpha}\right)+\partial_{\beta}\left(\rho u_{\alpha} u_{\beta}\right)=-\partial_{\alpha} p+\partial_{\beta}\left\{2 \mu S_{\alpha \beta}\right\}=-\partial_{\alpha} p+\mu \partial_{\beta} \partial_{\beta} u_{\alpha}
$$

For $\rho=$ constant, the incompressible Navier-Stokes equations are

$$
\begin{gathered}
\nabla \cdot \mathbf{u}=0 \\
\partial_{t} u_{\alpha}+\partial_{\beta}\left(u_{\alpha} u_{\beta}\right)=-\partial_{\alpha}\left(\frac{p}{\rho}\right)+\nu \partial_{\beta}^{2} u_{\alpha} .
\end{gathered}
$$

It is seen that Eq.(54) is exactly the same as the Narier-Stokes equation (56) in the incompressible limit, $\nabla \cdot \mathbf{u}=0$.

Collecting all coefficients so far, one obtains

$$
\begin{gathered}
A_{0}=\frac{4}{9} \rho, \quad A_{1}=\frac{1}{9} \rho, \quad A_{2}=\frac{1}{36} \rho, \\
B_{1}=\frac{1}{3} \rho, \quad B_{2}=\frac{1}{12} \rho, \\
C_{1}=\frac{1}{2} \rho, \quad C_{2}=\frac{1}{8} \rho .
\end{gathered}
$$

The remaining coefficients $D_{0}, D_{1}$ and $D_{2}$ are related by Eq. (13) and Eq. (33), so there is one free parameter. Since all coefficients of particle 2 are one-fourth of the corresponding 
coefficients of particle 1 , one can require $D_{1}=4 D_{2}$. Hence, the remaining coefficients are determined as

$$
D_{0}=-\frac{2}{3} \rho, \quad D_{1}=-\frac{1}{6} \rho, \quad D_{2}=-\frac{1}{24} \rho
$$

Finially, the equilibrium distribution functions are given as

$$
\begin{aligned}
f_{01}^{(0)} & =\frac{4}{9} \rho\left[1-\frac{3}{2} u^{2}\right], \\
f_{1 i}^{(0)} & =\frac{1}{9} \rho\left[1+3\left(\mathbf{e}_{1 i} \cdot \mathbf{u}\right)+\frac{9}{2}\left(\mathbf{e}_{1 i} \cdot \mathbf{u}\right)^{2}-\frac{3}{2} u^{2}\right], \\
f_{2 i}^{(0)} & =\frac{1}{36} \rho\left[1+3\left(\mathbf{e}_{2 i} \cdot \mathbf{u}\right)+\frac{9}{2}\left(\mathbf{e}_{2 i} \cdot \mathbf{u}\right)^{2}-\frac{3}{2} u^{2}\right] .
\end{aligned}
$$

